(2) Open Access Full Text Article

\title{
Higher serum total bilirubin predicts high risk of 3-year adverse outcomes in patients undergoing primary percutaneous coronary intervention
}

This article was published in the following Dove Press journal:

Therapeutics and Clinical Risk Management

\author{
Fan Gao' \\ Hua Qiang ${ }^{2}$ \\ Xiao-Juan Fan $^{2}$ \\ Qi Xue ${ }^{3}$ \\ Ling $\mathrm{Bai}^{2}$
}

'Clinical Research Center, The First Affiliated Hospital of Xi'an Jiaotong University, Xi'an, People's Republic of China; ${ }^{2}$ Department of Cardiovascular Medicine, The First Affiliated Hospital of Xi'an Jiaotong University, Xi'an, People's Republic of China; ${ }^{3}$ Institut des Sciences de la Terre, Centre National de la Recherche Scientifique, Saint-Martind'Hères, France
Correspondence: Ling Bai

Department of Cardiovascular Medicine,

The First Affiliated Hospital of Xi'an Jiaotong University, 277 Yanta West Road, Xi'an 71006I, People's Republic of China Tel +861 3759958590

Email bailing_xjtu@163.com
Purpose: Previous research findings on the association between serum total bilirubin (TB) and cardiovascular events varied with different study populations. Our objective was to clarify the association between serum TB at admission and long-term adverse outcomes in patients with acute coronary syndrome (ACS) and stable angina (SA) undergoing percutaneous coronary intervention (PCI).

Patients and methods: This prospective cohort study included 2,502 patients who underwent PCI. Information on the study population was obtained from the Dryad Digital Repository. The patients were divided into two groups: high $(>0.60 \mathrm{mg} / \mathrm{dL})$ and low $\mathrm{TB}$ groups $(\leq 0.60 \mathrm{mg} / \mathrm{dL})$ based on the optimal cutoff value achieved in the receiver operating characteristic curve analysis. The relationships between serum TB at admission and clinical outcomes after PCI were analyzed in multivariable logistic regression models and restricted cubic spline.

Results: In all patients undergoing PCI, TB $>0.60 \mathrm{mg} / \mathrm{dL}$ was associated with major adverse cardiovascular events (MACE) and cardiovascular death during a 3-year follow-up. The odds ratio (95\% confidence interval) was $1.60(1.22-2.10)$ and $1.81(1.22-2.70)$ for MACE and cardiovascular death, respectively. The association between TB and MACE was not altered by clinical presentation ( $p$ for interaction $=0.949$ ).

Conclusion: In patients with ACS and SA undergoing PCI, elevated serum TB was associated with increased risk of MACE and cardiovascular death.

Keywords: bilirubin, acute coronary syndrome, stable angina, primary percutaneous coronary intervention, major adverse cardiovascular; cerebrovascular events

\section{Introduction}

Serum bilirubin is a metabolic end product of heme, which partially reflects the activity of heme oxygenase (HO). ${ }^{1}$ In addition, bilirubin is a powerful antioxidant, which has been demonstrated to have a potential antioxidant capacity. ${ }^{2,3}$ The potential mechanism may be attributed to scavenging of reactive oxygen species. Bilirubin also has an antiinflammatory property based on the inhibition of endothelial adhesion molecules, such as intercellular adhesion molecule-1, vascular cell-adhesion molecule-1, and E-selectin, by tumor necrosis factor-alpha. ${ }^{4}$ The protective effects of serum total bilirubin (TB) against cardiovascular-related disease and adverse cardiovascular events have been reported in the last decade. Serum TB has been reported to exert a protective effect on hypertension, diabetes mellitus (DM), atherosclerosis, and coronary artery disease $(\mathrm{CAD}) .^{5-8} \mathrm{~A}$ meta-analysis containing a large population-based sample of 
7,222 participants reported that serum TB was inversely correlated with risk of cardiovascular diseases. ${ }^{9}$ Kim et al found that lower TB may be a predictor of adverse cardiovascular events in patients with $\mathrm{CAD}$ patients after primary percutaneous coronary intervention (PCI). ${ }^{10-12}$ In patients with hypertensive and acute syndrome $\mathrm{X}$, this inverse relationship between serum TB and adverse cardiovascular events remained. ${ }^{13-15}$ Conversely, recent studies found that serum TB concentration was elevated in patients with acute myocardial infarction (MI), and several cohort studies suggested that higher serum TB was associated with adverse outcomes in patients with MI. ${ }^{2,16-21}$ Based on bilirubin's anti-inflammatory and anti-oxidative properties, and different effects of serum TB on cardiovascular events in different populations, serum TB has become a heated research issue recently.

Although previous studies have evaluated the impact of the baseline serum TB on in-hospital cardiovascular outcomes after PCI, few studies have examined its influence on long-term follow-up outcomes. Furthermore, most previous studies focused on the relationship between serum TB and outcomes after PCI in patients with acute coronary syndrome (ACS), and limited evidence was available on patients with stable angina (SA). The clinical profiles and pathogenesis have been recognized to differ significantly between patients with ACS and SA. Its influence on clinical outcomes after PCI may also show differences. Our objective was to clarify the association between serum TB at admission and adverse cardiovascular events during follow up, particularly long-term clinical outcomes in patients with ACS and SA undergoing PCI.

\section{Materials and methods}

\section{Population}

Study data were acquired from the Dryad Digital Repository. Our data are accessible in the Digital Repository (https://datadryad.org/resource/doi:10.5061/ dryad.13d31). All patients were collected from a single high-volume PCI center who performed successful PCI with at least one drug-eluting stent (DES) from July 2009 to August 2011. Full details of the study population and PCI procedures have been reported previously. ${ }^{22,23}$ Patients in this study were excluded based on the following criteria: (1) age $<18$ years; (2) patients with serum TB at admission $>2.0 \mathrm{mg} / \mathrm{dL}$; and (3) missing covariates or follow-up information. The study was approved by the Ethics Committee of the First Affiliated Hospital of Zhengzhou University with a waiver of informed consent. Data were analyzed anonymously in this study.

\section{Baseline data collection}

All basic clinical characteristics information was collected at admission, including demographic information, cardiovascular risk factors, medication at discharge, and angiographic and procedural information. Smoking was defined as patients who smoked within the previous 10 years. Diabetes mellitus (DM) was defined as a fasting plasma glucose concentration $\geq 126 \mathrm{mg} / \mathrm{dL}$ or current treatment with hypoglycemic agents. Hypertension was defined as a systolic blood pressure $\geq 140 \mathrm{mmHg}$ or diastolic blood pressure $\geq 90 \mathrm{mmHg}$ or use of antihypertensive drugs. Other disease histories were self-reported. Cardiogenic shock was defined as obvious and persistent hypotension (>30 min) with systolic arterial pressure $<80 \mathrm{mmHg}$ with signs of hypoperfusion due to the dysfunction of the ventricles of the heart. ${ }^{22}$

\section{Clinical outcomes}

The primary endpoint was a long-term major adverse cardiovascular event (MACE) during a 3-year follow-up. The secondary endpoints included in-hospital and followup outcomes. In in-hospital outcomes, cardiovascular death, and nonfatal MI were observed. In long-term adverse outcomes, cardiovascular death, nonfatal MI, target vessel revascularization (TVR), any revascularization, in-stent restenosis, angina, and stroke were observed. MACE was a composite endpoint, defined as cardiovascular death, nonfatal MI, and stroke occurrence. All followup information after discharge of recruited patients was obtained through patient visits to the outpatient clinic or readmission or via a telephone interview with patients and their families. Outcome data were entered by trained research personnel, and the occurrence of adverse events was adjudicated by an independent committee not involved in the procedures.

\section{Statistical analysis}

All patients were divided into two groups: the high $(>0.60 \mathrm{mg} / \mathrm{dL})$ and low TB groups $(\leq 0.60 \mathrm{mg} / \mathrm{dL})$. The optimal cutoff point $(0.60 \mathrm{mg} / \mathrm{dL})$ was determined by receiver operating characteristic curve analysis. Normally distributed, continuous variables were presented as means (standard deviation), and non-normally distributed data were shown as medians [inter-quartile ranges]. Categorical variables were shown as percentages. Student $t$-test or Mann-Whitney 
$\mathrm{U}$ test were used to compare continuous variables in two groups. Chi-squared test or Fisher exact test were applied for analyzing the differences in categorical data. Multiple linear regression analysis was used to determine the relationship of serum TB with cardiovascular risk factors, including age, sex, DM, hypertension, heart rate, smoking, atrial fibrillation, heart failure, history of MI, multiple diseased vessels, lesion of left main stem, restenosis lesions, and ACS. The regression coefficient and 95\% CI were depicted.

The associations of serum TB with cardiovascular events after PCI were assessed using multivariable logistic regression analysis with adjustment sets as follows: 1) unadjusted; 2) ageand sex-adjusted; and 3) multivariable: additionally adjusted for DM, hypertension, heart rate, smoking, atrial fibrillation, heart failure, history of MI, multiple diseased vessels, lesion of left main stem, restenosis lesions, ACS, triglycerides, statin, and $\beta$-blocker. To assess the shape of the association between TB and MACE, we introduced terms with common transformations of TB inclusive of linear, quadratic, logarithmic, categorical (tertiles), and binary (groups as specified above) in multivariable models and used nonparametric restricted cubic splines with four knots defined as the 5th, 35th, 65th, and 95th percentiles of the distribution of TB. The reference value for TB was $0.52 \mathrm{mg} / \mathrm{dL}$ (the median value of TB).

We also examined for interaction the effect of TB on various outcomes by clinical presentation (ACS and SA) in the multivariable model and explored the relationship between serum TB and various follow-up outcomes after PCI in two subgroups (ACS and SA) in logistic regression models after adjusting for age, sex, DM, and hypertension. A two-tailed $p \leq 0.05$ was considered statistically significant. Statistical analysis was conducted using SPSS 24.0 (IBM, Armonk, NY, USA) and SAS software 9.3 (SAS Institute Inc., Cary, NC, USA).

\section{Results}

\section{Baseline characteristics}

Of 2,533 patients who received successful PCI from July 2009 to August 2011, 31 patients were excluded based on the exclusion criteria. Finally, 2,502 patients were enrolled in this study (Figure 1). The mean age was 59.9 \pm 11.1 years, and $68.0 \%$ of the patients were men. Clinical, laboratory, and angiographic information in the total population are listed in Table 1. The mean serum TB level at admission in the high $(>0.60 \mathrm{mg} / \mathrm{dL})$ and low TB groups $(\leq 0.60 \mathrm{mg} / \mathrm{dL})$ was $0.70 \mathrm{mg} / \mathrm{dL}[0.60-0.90]$ and $0.40 \mathrm{mg} / \mathrm{dL}$ [0.30-0.50], respectively. Furthermore, 980 patients $(39.2 \%)$ had serum TB $>0.60 \mathrm{mg} / \mathrm{dL}$. The high TB group had higher initial systolic, diastolic blood pressure, heart rate, fasting blood sugar, creatinine, and uric acid level, and lower triglycerides and left ventricular ejection fraction than the low TB group. In addition, the high TB group tended to have more male patients, a higher percentage of urgent PCI, more comorbidities, including previous coronary artery bypass graft (CABG), atrial fibrillation, heart failure, and more complex lesions, including bifurcation lesions and ostial lesions compared with the low TB group.

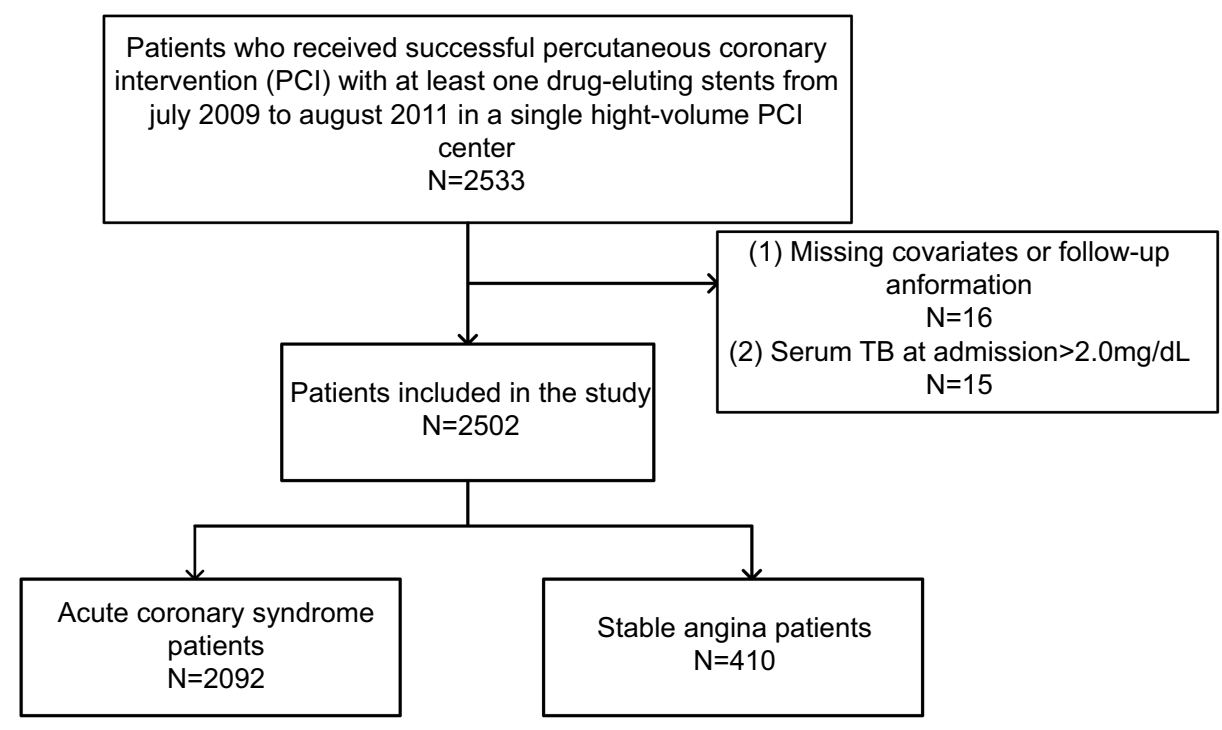

Figure I Study population selection.

Abbreviation: TB, total bilirubin. 
Table I Clinical information, laboratory tests and procedural characteristics

\begin{tabular}{|c|c|c|c|}
\hline \multirow[t]{2}{*}{ Variable } & \multirow{2}{*}{$\begin{array}{l}T B>0.60 \mathrm{mg} / \mathrm{dL} \\
\mathrm{n}=980\end{array}$} & \multirow{2}{*}{$\begin{array}{l}\mathrm{TB} \leq 0.60 \mathrm{mg} / \mathrm{dL} \\
\mathrm{n}=\mathrm{I}, 522\end{array}$} & \multirow[t]{2}{*}{$p$-value } \\
\hline & & & \\
\hline Age (years) & $59.7 \pm 11.2$ & $60.1 \pm 11.0$ & 0.258 \\
\hline Male, $n(\%)$ & $764(78)$ & $938(61.6)$ & $<0.001$ \\
\hline \multicolumn{4}{|l|}{ Admission characteristics } \\
\hline Systolic BP (mmHg) & $107 \pm 28.3$ & $100.8 \pm 27.9$ & $<0.001$ \\
\hline Diastolic BP (mmHg) & $78.1 \pm 12.0$ & $76.6 \pm 11.6$ & $<0.001$ \\
\hline Urgent $\mathrm{PCl}, n(\%)$ & $57(5.8)$ & $40(2.6)$ & $<0.001$ \\
\hline Cardiogenic shock, n (\%) & $3(0.3)$ & $\mathrm{I}(0.1)$ & 0.142 \\
\hline Heart rate (/min) & $72.9 \pm 11.4$ & $71.6 \pm 10.8$ & 0.022 \\
\hline \multicolumn{4}{|l|}{ Laboratory test } \\
\hline Fasting glycemia (mmol/L) & $6.1 \pm 3$ & $6 \pm 3$ & 0.004 \\
\hline Creatinine (mmol/L) & $73 \pm 24.1$ & $72.2 \pm 37$ & 0.001 \\
\hline Uric acid (mmol/L) & $309.4 \pm 95.2$ & $299 \pm 83.9$ & 0.007 \\
\hline TB $(\mathrm{mg} / \mathrm{dL}), \operatorname{median}[\mathrm{IQR}]$ & $0.7[0.6-0.9]$ & $0.4[0.3-0.5]$ & $<0.001$ \\
\hline Total cholesterol (mmol/L) & $4.2 \pm 1$ & $4.3 \pm 1.1$ & 0.145 \\
\hline Triglycerides (mmol/L) & $1.8 \pm 1$ & $2 \pm 1.4$ & 0.005 \\
\hline HDL-C (mmol/L) & $I . I \pm 0.3$ & $1.1 \pm 0.3$ & 0.446 \\
\hline LDL-C (mmol/L) & $2.7 \pm 0.8$ & $2.7 \pm 0.9$ & 0.100 \\
\hline \multicolumn{4}{|l|}{ History of disease, $n(\%)$} \\
\hline Stroke & $5 \mid(5.2)$ & $82(5.4)$ & 0.842 \\
\hline Peripheral vascular disease & $4(0.4)$ & $2(0.1)$ & 0.167 \\
\hline Previous $\mathrm{PCl}$ & $64(6.5)$ & $105(6.9)$ & 0.720 \\
\hline Previous CABG & I4 (I.4) & $6(0.4)$ & 0.005 \\
\hline Myocardial infarction & $103(10.5)$ & $129(8.5)$ & 0.087 \\
\hline COPD & II (I.I) & II (0.7) & 0.314 \\
\hline Atrial fibrillation & $28(2.9)$ & $21(1.4)$ & 0.015 \\
\hline Heart failure & $124(12.7)$ & $169(11.1)$ & 0.030 \\
\hline \multicolumn{4}{|l|}{ Risk factors, $n(\%)$} \\
\hline Hypertension & $449(45.8)$ & $780(5 \mathrm{I} .2)$ & 0.008 \\
\hline Diabetes mellitus & $182(18.6)$ & $333(21.9)$ & 0.046 \\
\hline Smoking & $334(34.1)$ & $475(31.2)$ & 0.134 \\
\hline \multicolumn{4}{|l|}{ Medication, $n$ (\%) } \\
\hline Aspirin & 967 (98.7) & I,502 (98.7) & 0.640 \\
\hline Clopidogrel & $943(96.2)$ & $\mathrm{I}, 460(95.9)$ & 0.549 \\
\hline$\beta$-blocker & $638(65.1)$ & $1,060(69.6)$ & 0.018 \\
\hline ACEI & $506(5 I .6)$ & $835(54.9)$ & 0.483 \\
\hline $\mathrm{CCB}$ & $201(20.5)$ & $390(25.6)$ & 0.003 \\
\hline Statin & $864(88.2)$ & I,4II (92.7) & $<0.001$ \\
\hline \multicolumn{4}{|c|}{ Angiographic and procedural characteristics } \\
\hline Total stent length $(\mathrm{mm})$ & $48.6 \pm 30.6$ & $51.3 \pm 33.9$ & 0.277 \\
\hline Stent diameter $(\mathrm{mm})$ & $3.1 \pm 1.3$ & $3.1 \pm 0.4$ & 0.056 \\
\hline Stent type, $n(\%)$ & & & 0.760 \\
\hline SES & $630(64.3)$ & $997(65.5)$ & \\
\hline PES & 202(20.6) & $296(19.5)$ & \\
\hline Mixture & $148(15.1)$ & $229(15.1)$ & \\
\hline 3 or more diseased vessels, $n(\%)$ & $231(23.5)$ & $366(24.0)$ & 0.749 \\
\hline
\end{tabular}

(Continued) 
Table I (Continued).

\begin{tabular}{|c|c|c|c|}
\hline \multirow[t]{2}{*}{ Variable } & $T B>0.60 \mathrm{mg} / \mathrm{dL}$ & $\mathrm{TB} \leq 0.60 \mathrm{mg} / \mathrm{dL}$ & \multirow[t]{2}{*}{$p$-value } \\
\hline & $n=980$ & $n=1,522$ & \\
\hline \multicolumn{4}{|l|}{ Location of target lesions, $n(\%)$} \\
\hline Left main stem & $32(3.3)$ & $52(3.4)$ & 0.331 \\
\hline LAD & $823(84)$ & I,246 (8I.9) & 0.063 \\
\hline LCX & $476(48.6)$ & $736(48.4)$ & 0.539 \\
\hline RCA & $475(48.5)$ & $761(50)$ & 0.326 \\
\hline Coronary bypass graft & $2(0.2)$ & $2(0.1)$ & 0.180 \\
\hline Total chronic occlusions, $n$ (\%) & $143(14.6)$ & $182(12)$ & 0.056 \\
\hline Ostial lesions, $n(\%)$ & $129(13.2)$ & $145(9.5)$ & 0.004 \\
\hline Bifurcation lesion, $n(\%)$ & $156(15.9)$ & $290(19.1)$ & 0.045 \\
\hline Restenotic lesion, $n(\%)$ & $13(1.3)$ & $21(1.4)$ & 0.839 \\
\hline LVEF (\%) & $60.4(8.5)$ & $61.1(6.8)$ & 0.783 \\
\hline Type of patients & & & 0.018 \\
\hline ACS & $798(81.4)$ & I,294 (85) & \\
\hline SA & $182(18.6)$ & $228(15)$ & \\
\hline
\end{tabular}

Abbreviations: ACS, acute coronary syndrome; BP, blood pressure; CABG, coronary artery bypass graft; HDL-C, high-density lipoprotein cholesterol; LDL-C, low-density lipoprotein cholesterol; $\mathrm{PCl}$, percutaneous coronary intervention; SA, stable angina; TB, total bilirubin.

\section{In-hospital and follow-up clinical outcomes}

Clinical outcomes following PCI in the total population are listed in Table 2. Over the follow-up period (median, 29.8 months, quartiles, 25.6-34 months); 250 patients (10.0\%) experienced MACE. Among them, 114 patients (4.6\%) resulted in cardiovascular death, and 117 (4.7\%) and $38(1.5 \%)$ patients experienced nonfatal MI and stroke, respectively. The majority of MACE was attributed to nonfatal MI (117 events, 4.6\%) and cardiovascular death (114 events, $4.6 \%)$. The rates of MACE (12.8\% vs $8.2 \%, p<0.001)$ and cardiovascular death $(6.3 \%$ vs $3.4 \%$, $p<0.001)$ in the high TB group were higher than those in the low TB group. With regard to nonfatal MI, TVR, any revascularization, in-stent restenosis, angina, stroke, and all three in-hospital outcomes, no significant differences were found between the high and low TB groups. Clinical outcomes following PCI in ACS and SA patients separately are listed in Table S1.

\section{Covariates of serum TB}

In multiple linear regression analysis, serum TB at admission had positive correlation with age $(\beta=0.001$ and $95 \%$ CI: $0.0002-0.002)$, male sex $(\beta=0.078$ and $95 \% \mathrm{CI}$ : $0.051-0.104)$, hypertension $(\beta=-0.024,95 \% \mathrm{CI}:-0.046$ to $-0.003)$, atrial fibrillation $(\beta=0.124,95 \% \mathrm{CI}: 0.047-0.202)$, heart failure $(\beta=0.072,95 \% \mathrm{CI}: 0.039-0.105$; all $p$-values $<0.05)$; see Table 3.

\section{Association between TB and clinical outcomes}

Associations between serum TB at admission and clinical outcomes following PCI in the total population are listed in Table 4. In follow-up outcomes, serum TB was associated with MACE and cardiovascular death in univariate logistic regression analysis (MACE: OR and 95\% CI, 1.63 [1.26-2.12], $p<0.001$; cardiovascular death: OR and 95\% CI, 1.91 [1.31-2.79], $p=0.009$ ), and age- and sex-adjusted logistic regression analysis (MACE: OR and 95\% CI, 1.60 [1.23-2.09], $p<0.001$; cardiovascular death: OR and $95 \%$ CI, 1.94 [1.32-2.86], $p=0.014)$. This association remained in MACE and cardiovascular death after further adjustment for other cardiovascular risk factors (MACE: OR and 95\% CI, 1.60[1.2-2.10], $p<0.001$; cardiovascular death: OR and $95 \%$ CI, 1.81 [1.22-2.70], $p=0.003$ ). Serum TB did not show any significant relationship with respect to other clinical outcomes. Besides, after transformation of TB, relationships of linear, quadratic, logarithmic, and categorical (tertiles) TB with MACE in fully adjusted models are shown in Figure 2. When TB was a categorical variable, a positive association was observed ( $\mathrm{OR}=0.94,95 \% \mathrm{CI}$ : $0.56-1.59$ for tertile 2 vs tertile 1 ; OR $=1.44,95 \%$ CI: $0.89-2.34$ for tertile 3 vs tertile 1). When TB was a continuous, logarithmic, or quadratic variable, a positive association was also observed (Figure 2). The dose-response relationship between serum TB and MACE is given in Table 5. Restricted cubic spline is 
Table 2 In-hospital and follow-up outcomes in total population

\begin{tabular}{|c|c|c|c|}
\hline \multirow[t]{2}{*}{ Clinical outcomes } & $\mathrm{TB}>0.60 \mathrm{mg} / \mathrm{dL}$ & $\mathrm{TB} \leq 0.60 \mathrm{mg} / \mathrm{dL}$ & \multirow[t]{2}{*}{$p$-value } \\
\hline & $n=980$ & $n=1,522$ & \\
\hline \multicolumn{4}{|c|}{ In-hospital outcomes, n (\%) } \\
\hline Cardiovascular death & II (I.I) & $7(0.5)$ & 0.055 \\
\hline Nonfatal MI & $6(0.6)$ & $9(0.6)$ & 0.947 \\
\hline MACE & $16(1.6)$ & $15(1)$ & 0.153 \\
\hline \multicolumn{4}{|c|}{ Follow-up outcomes, n (\%) } \\
\hline MACE & $125(12.8)$ & $125(8.2)$ & $<0.001$ \\
\hline Cardiovascular death & $62(6.3)$ & $52(3.4)$ & $<0.001$ \\
\hline Nonfatal MI & $55(5.6)$ & $62(4.1)$ & 0.075 \\
\hline TVR & $57(5.8)$ & $66(4.3)$ & 0.095 \\
\hline Any revascularization & $85(8.7)$ & $114(7.5)$ & 0.286 \\
\hline In-stent restenosis & $62(6.3)$ & $83(5.5)$ & 0.362 \\
\hline Angina & $112(11.4)$ & $175(11.5)$ & 0.958 \\
\hline Stroke & $17(1.7)$ & $21(1.4)$ & 0.479 \\
\hline
\end{tabular}

Abbreviations: MACE, major adverse cardiovascular events; MI, myocardial infarction; TB, total bilirubin; TVR, target vessel revascularization.

Table 3 Factors related to TB by multiple linear regression analysis

\begin{tabular}{|l|l|l|}
\hline Parameters & $\boldsymbol{\beta}(\mathbf{9 5} \% \mathbf{C I})$ & $\boldsymbol{p}$-value \\
\hline Age (years) & $0.001(0.0002-0.002)$ & 0.012 \\
Male & $0.078(0.05 \mathrm{I}-0.104)$ & $<0.00 \mathrm{I}$ \\
Diabetes mellitus & $-0.015(-0.042-0.0 \mathrm{II})$ & 0.263 \\
Hypertension & $-0.024(-0.046$ to- & 0.027 \\
& $0.003)$ & \\
Smoking & $-0.024(-0.050-0.00 \mathrm{I})$ & 0.063 \\
Atrial fibrillation & $0.124(0.047-0.202)$ & 0.002 \\
Heart failure & $0.072(0.039-0.105)$ & $<0.00 \mathrm{I}$ \\
Myocardial infarction & $0.021(-0.019-0.06 \mathrm{I})$ & 0.307 \\
history & & \\
3 or more diseased vessels & $-0.016(-0.04 \mathrm{I}-0.009)$ & 0.215 \\
Lesion of left main stem & $-0.048(-0.107-0.01 \mathrm{I})$ & 0.114 \\
Restenosis lesions & $-0.018(-0.109-0.074)$ & 0.705 \\
Heart rate (/min) & $0.002(0.0005-0.003)$ & 0.002 \\
ACS & $0.008(-0.023-0.040)$ & 0.605 \\
\hline
\end{tabular}

Abbreviations: ACS, acute coronary syndrome; TB, total bilirubin.

shown in Figure 3, and the risk of MACE increased with an increase of TB ( $p$ overall $=0.025)$. A nonlinear doseresponse relationship between $\mathrm{TB}$ and MACE was not found ( $p$ nonlinear $=0.208$ ).

The association between serum TB at admission and follow-up adverse events in patients undergoing PCI was analyzed separately in patients with ACS and SA (Figure 4). In subgroup analysis, higher TB was associated with a greater risk of MACE (OR and 95\% CI, 1.61 [1.20-2.17], $p<0.001)$ and cardiovascular death (OR and 95\% CI, 1.89 [1.22-2.93]). Although patients with SA did not show any significant relationship between serum TB and outcomes, no significant interaction was detected in any outcomes (all $p$ interaction $>0.05$ ).

\section{Discussion}

The main finding of the present study was that serum TB level at admission was related with MACE and cardiovascular death during a 3-year follow-up with adjustment for age, sex, DM, hypertension, heart rate, smoking, atrial fibrillation, heart failure, history of MI, multiple diseased vessels, lesion of left main stem, restenosis lesions, and acute coronary syndrome. Our study result clearly showed that a high baseline serum TB level was a simple and powerful predictor of long-term MACE and cardiovascular death in patients undergoing PCI regardless of clinical presentation of CAD.

Previous literature demonstrated that increased serum TB may be protective against adverse vascular events under normal condition. . $3,9-11,13,14,24-26^{\text {Conversely, }}$ recent reports found that initial serum $T B$ was a prognostic marker of no-reflow and in-hospital adverse outcomes in patients with ST elevation myocardial infarction (STEMI) and MI undergoing PCI. ${ }^{2,17-19,21,27}$ Sahin et al found that patients with STEMI with higher TB level accumulated in the high SYNTAX score group than the low SYNTAX score group. ${ }^{28}$ The results involving TB and cardiovascular outcomes may be different between emergent and non-emergent patients, and most studies observed in-hospital outcomes in patients with ACS rather than patients with SA. Therefore, the present study might 
Table 4 Associations between total bilirubin $>0.60 \mathrm{mg} / \mathrm{dL}$ and clinical outcomes in total population

\begin{tabular}{|l|l|l|l|}
\hline Clinical outcomes & Model I & Model 2 & Model 3 \\
\cline { 2 - 4 } & OR (95\%Cl) & OR (95\%CI) & OR (95\%CI) \\
\hline $\begin{array}{l}\text { In-hospital outcomes } \\
\text { Cardiovascular death }\end{array}$ & $2.46(0.95-6.36)$ & $2.64(1.00-6.93)$ & NA \\
Nonfatal MI & $1.04(0.37-2.92)$ & $1.10(0.38-3.14)$ & NA \\
MACE & $1.67(0.82-3.39)$ & $1.73(0.84-3.55)$ & NA \\
\hline Follow-up outcomes & & & \\
MACE & $1.63(1.26-2.12)$ & $1.60(1.23-2.09)$ & $1.60(1.22-2.10)$ \\
Cardiovascular death & $1.91(1.31-2.79)$ & $1.94(1.32-2.86)$ & $1.81(1.22-2.70)$ \\
Nonfatal MI & $1.40(0.97-2.03)$ & $1.38(0.95-2.02)$ & $1.40(0.96-2.06)$ \\
TVR & $1.36(0.95-1.96)$ & $1.60(1.23-2.09)$ & $1.26(0.86-1.85)$ \\
Any revascularization & $1.17(0.88-1.57)$ & $1.09(0.8 I-1.47)$ & $1.12(0.83-1.5 \mathrm{I})$ \\
In-stent restenosis & $1.17(0.83-1.64)$ & $1.12(0.79-1.58)$ & $1.15(0.8-1.64)$ \\
Angina & $0.99(0.77-1.28)$ & $0.99(0.77-1.28)$ & $1.01(0.78-1.31)$ \\
\hline
\end{tabular}

Abbreviations: MACE, major adverse cardiovascular events; MI, myocardial infarction; TVR, target vessel revascularization.

A

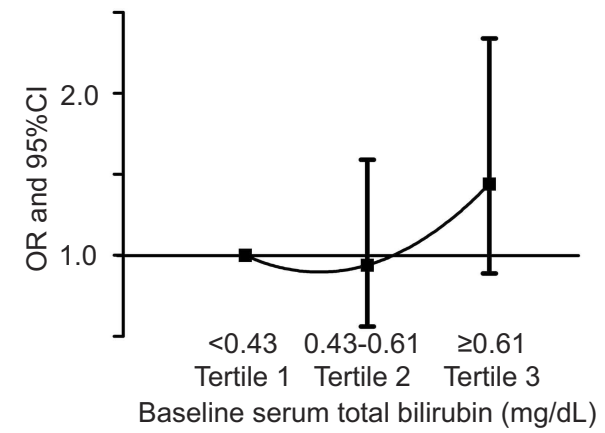

B

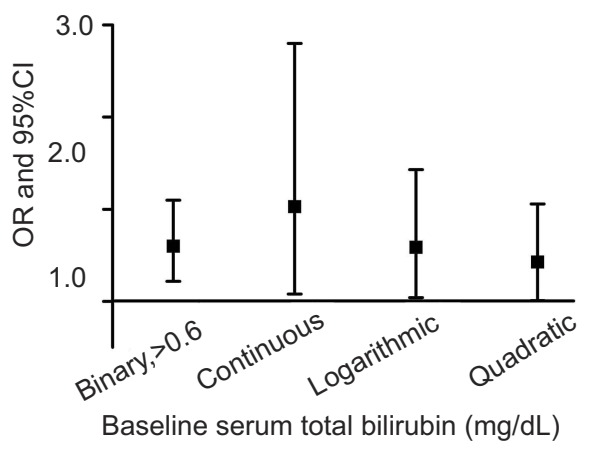

Figure 2 Correlation of total bilirubin (TB) with major adverse cardiovascular and cerebrovascular events. (A) Categorical TB with tertiles; (B) binary, continuous, logarithmic and quadratic TB.

Table 5 Dose-response relationship between total serum bilirubin and long-term MACE

\begin{tabular}{|l|l|l|}
\hline \multirow{2}{*}{ Serum total bilirubin (mg/dL) } & \multicolumn{2}{l|}{ MACE } \\
\cline { 2 - 3 } & OR & $\mathbf{9 5 \%} \mathbf{~ C l}$ \\
\hline 0.21 & 0.81 & $0.50-1.33$ \\
\hline 0.44 & 0.88 & $0.79-0.97$ \\
\hline 0.52 & \multicolumn{3}{|l|}{1.0 (Reference) } \\
\hline 0.6 & 1.16 & $1.01-1.32$ \\
\hline 1.06 & 1.41 & $1.01-1.97$ \\
\hline$P$ overall & 0.025 \\
\hline$P$ nonlinear & 0.208 \\
\hline
\end{tabular}

Abbreviation: MACE, major adverse cardiovascular events. provide evidence to fully evaluate the association between serum TB and outcomes over a 3-year follow-up in patients with ACS and SA.

One conceivable reason to explain the positive relationship between TB and long-term outcomes after PCI is that higher serum TB level at baseline may be a reflection of more severe disease status. It can be postulated that the elevation of bilirubin may result from inadequate arterial perfusion of liver and hepatic congestion owing to cardiac dysfunction. ${ }^{21,27}$ Our finding supported this hypothesis because we observed that patients with higher bilirubin concentration tended to be older and had worse co-morbidities, including atrial fibrillation and heart failure, which may cause higher possibility of long-term dismal outcomes after PCI. In addition, recent finding from Htun et al suggested that elevated bilirubin may be a surrogate 


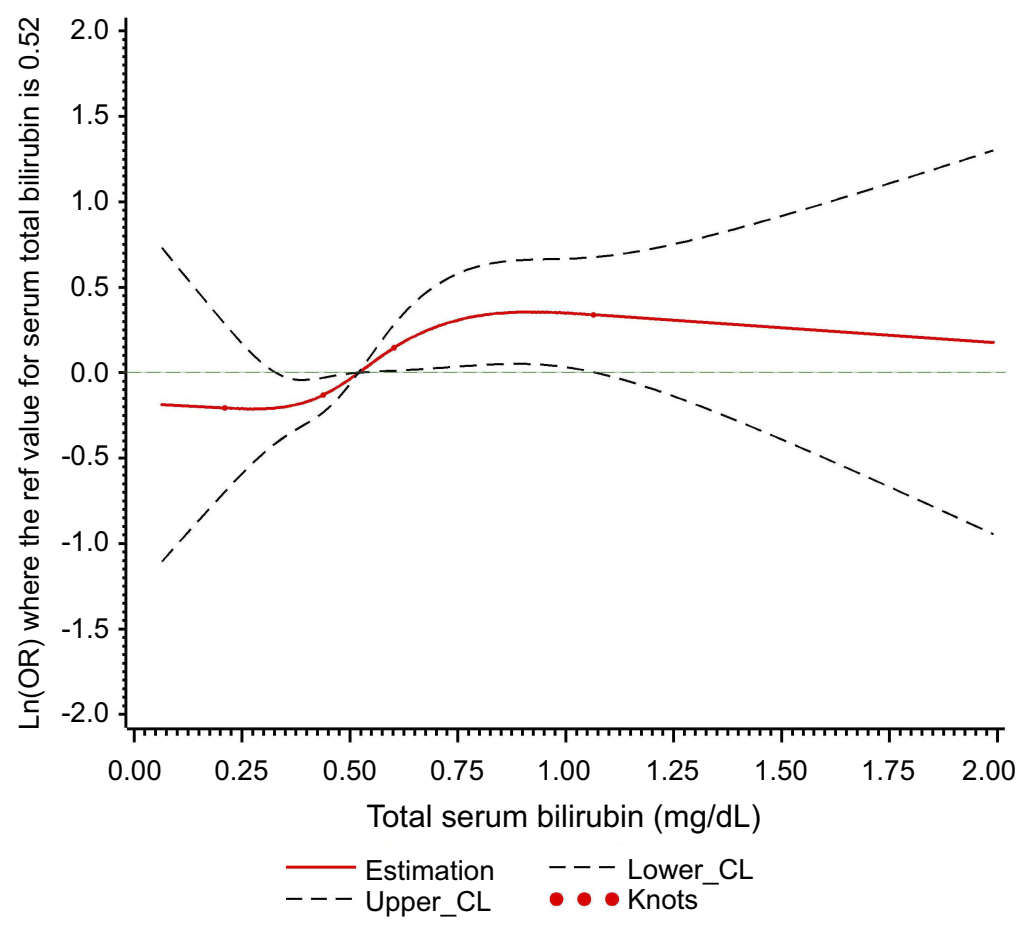

Figure 3 Plot of dose-response relationship between serum total bilirubin and MACE during follow-up ( $p$ nonlinear $=0.208, p$ overall=0.025). Abbreviations: $\mathrm{CL}$, confidence limit; MACE, major adverse cardiovascular events.

marker of unstable plaques, which may cause MI, stroke, and death. $^{29}$

Another hypothesis that may explain the relationship between serum TB and unfavorable cardiovascular outcomes in patients with ACS is acute stress reaction. An inducible isoform in response to diverse cellular stress, such as oxidative stress, hypoxia, heavy metals, cytokines, and heme oxygenase 1 (HO-1), reflects the extent of inflammatory reaction to myocardial damage. In previous reports, HO-1 was found to be notably upregulated in the myocardium under infarction and pressure overload condition in animal models. ${ }^{30}$ The study by Okuhara et al demonstrated a positive correlation between $\mathrm{HO}-1$ protein and its end product bilirubin. ${ }^{31}$ Amanvermez et al also found that oxidative end products in the first hour of patients with ACS were higher than those in the healthy population. ${ }^{32}$ Therefore, higher serum TB concentration may also represent more serious myocardial damage in the acute period and following poor outcomes. Acute stress reaction may cause an evident correlation between TB and clinical outcomes in patients with ACS. In patients with SA, we also observed that patients with higher TB tended to have a higher risk of MACE, cardiovascular death, and nonfatal MI, although no statistical significance was observed, which may be due to the smaller sample size of patients with SA. In patients with SA, the potential positive relationship between $\mathrm{TB}$ and MACE may be attributed to TB as a marker of more severe disease or more severe cardiovascular dysfunction.

Some limitations still need to be considered. First, only serum TB value at admission was assessed in the study. There was no information regarding the dynamic alteration of serum TB level and time points of adverse event occurrence. Confirmation of the time-dependent association between serum TB level and prognosis is impossible. Second, HO-1 and other markers related to oxidative stress were not measured. ${ }^{18}$ Third, follow-up information obtained through a telephone interview may be an important bias in the data collection. Finally, although multivariable logistic regression analyses were performed, some potential confounding factors may not be adjusted for in our analyses because of the limited sample size.

\section{Conclusion}

We conclude that in patients with ACS and SA who underwent PCI, elevated serum TB level was associated with a greater risk of MACE and cardiovascular death. Baseline serum TB may be treated as an indicator of poor outcome after PCI regardless of whether they are patients with ACS 
Follow-up outcomes

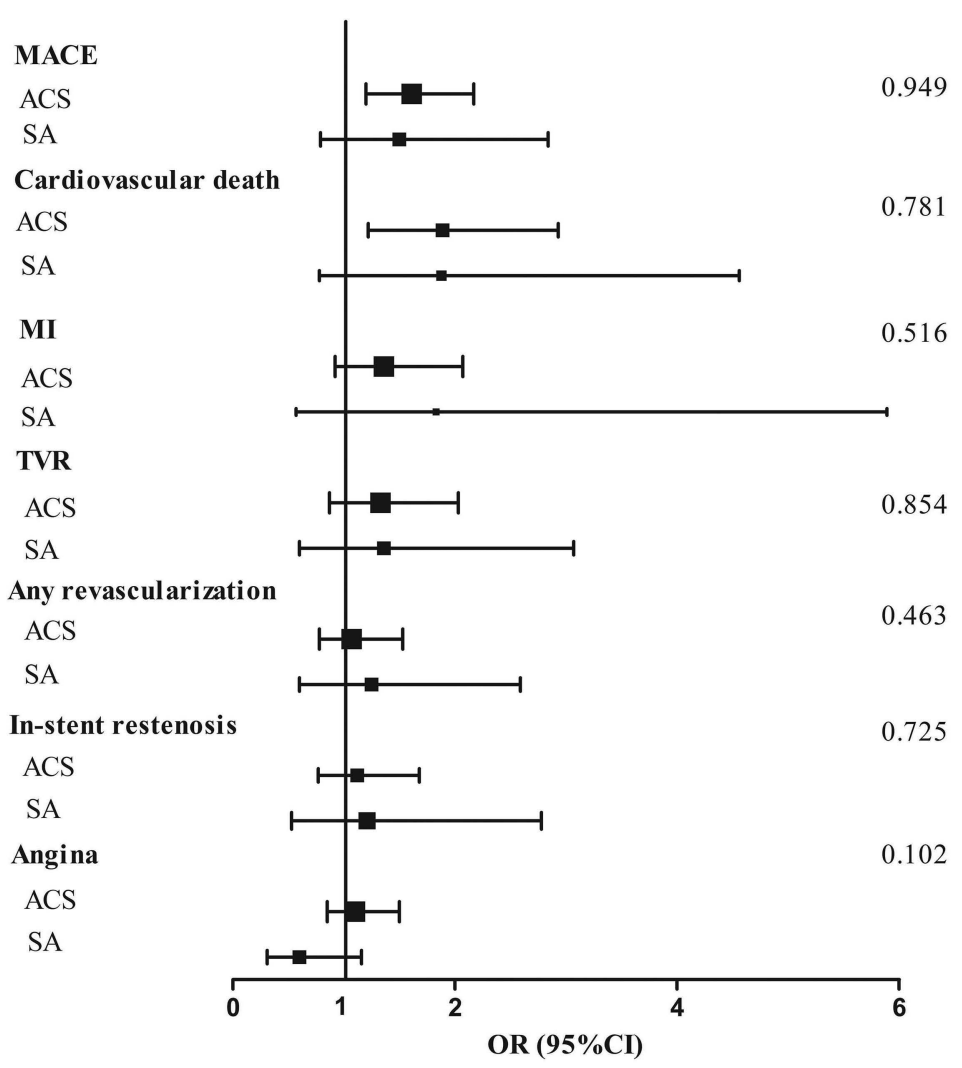

Figure 4 Associations between total bilirubin $>0.60 \mathrm{mg} / \mathrm{dL}$ and clinical outcomes in ACS and SA patients.

Abbreviations: ACS, acute coronary syndrome; MACE, major adverse cardiovascular events; MI, myocardial infarction; SA, stable angina; TVR, target vessel revascularization. Logistic regression model adjusted for age, sex, diabetes mellitus and hypertension.

or SA. Our findings warrant additional research, particularly in a larger prospective cohort study, to assess the impact of serum TB on long-term adverse cardiovascular events.

\section{Acknowledgments}

This work was supported by the Clinical Research Award of the First Affiliated Hospital of Xi'an Jiaotong University, China (No. XJTU1AF-CRF-2018-008).

\section{Author contributions}

FG: designing the study, data management, analysis, and drafting the manuscript. XJF, HQ and QX: data analysis and interpretation of results. LB: design of the study and paper revision. All authors contributed to data analysis, drafting or revising the article, gave final approval of the version to be published, and agree to be accountable for all aspects of the work.

\section{Disclosure}

The authors report no conflicts of interest in this work.

\section{References}

1. Mahabadi AA, Lehmann N, Mohlenkamp S, et al. Association of bilirubin with coronary artery calcification and cardiovascular events in the general population without known liver disease: the Heinz Nixdorf Recall study. Clin Res Cardiol. 2014;103(8):647-653. doi:10.1007/s00392-014-0697-z

2. Chung SR, Yang TH, Shin HC, et al. Initial total bilirubin and clinical outcome in patients with ST-segment elevation myocardial infarction undergoing primary percutaneous coronary intervention with drug-eluting stents. Circ J. 2016;80(6):1437-1444. doi:10.1253/circj. CJ-15-1397

3. Ajja R, Lee DC, Sui X, Church TS, Steven NB. Usefulness of serum bilirubin and cardiorespiratory fitness as predictors of mortality in men. Am J Cardiol. 2011;108(10):1438-1442. doi:10.1016/j. amjcard.2011.06.067

4. Nishimura T, Tanaka M, Saisho Y, Miyakoshi K, Tanaka M, Itoh H. Lower serum total bilirubin concentration is associated with higher prevalence of gestational diabetes mellitus in Japanese pregnant women. Endocr J. 2018;65(12):1199-1208. doi:10.1507/endocrj.EJ17-0533

5. Chin HJ, Song YR, Kim HS, et al. The bilirubin level is negatively correlated with the incidence of hypertension in normotensive Korean population. J Korean Med Sci. 2009;24:S50-S56. doi:10.3346/ jkms.2009.24.S1.S50

6. Han SS, Na KY, Chae DW, Kim YS, Kim S, Chin HJ. High serum bilirubin is associated with the reduced risk of diabetes mellitus and diabetic nephropathy. Tohoku J Exp Med. 2010;221(2):133-140. 
7. Hopkins PN, Wu LL, Hunt SC, James BC, Vincent GM, Williams RR. Higher serum bilirubin is associated with decreased risk for early familial coronary artery disease. Arter Thromb Vasc Biol. 1996;16(2):250-255. doi:10.1161/01.ATV.16.2.250

8. Schwertner HA, Jackson WG, Tolan G. Association of low serum concentration of bilirubin with increased risk of coronary-artery disease. Clin Chem. 1994;40(1):18-23.

9. Kunutsor SK, Bakker SJ, Gansevoort RT, Chowdhury R, Dullaart RP. Circulating total bilirubin and risk of incident cardiovascular disease in the general population. Arterioscler Thromb Vasc Biol. 2015;35 (3):716-724. doi:10.1161/ATVBAHA.114.304929

10. Kim HW, Choi DH, Lim L, et al. Usefulness of serum bilirubin levels as a biomarker for long-term clinical outcomes after percutaneous coronary intervention. Heart Vessels. 2015;30(6):728-733. doi:10.1007/s00380-014-0546-Z

11. Zhang MM, Gao Y, Zheng YY, et al. Association of fasting serum bilirubin levels with clinical outcomes after percutaneous coronary intervention: a prospective study. Cardiovasc Toxicol. 2017;17 (4):471-477. doi:10.1007/s12012-017-9405-3

12. Wei S, Mao L, Liu B, Zhong L. Serum biomarkers and the prognosis of AMI patients. Herz. 2014;39(3):384-389. doi:10.1007/s00059013-3828-9

13. Huang SS, Huang PH, Leu HB, Wu T-C, Lin S-J, Chen J-W. Serum bilirubin predicts long-term clinical outcomes in patients with cardiac syndrome X. Heart. 2010;96(15):1227-1232. doi:10.1136/ hrt.2009.192393

14. McCallum L, Panniyammakal J, Hastie CE, et al. Longitudinal blood pressure control, long-term mortality, and predictive utility of serum liver enzymes and bilirubin in hypertensive patients. Hypertension. 2015;66(1):37-43. doi:10.1161/HYPERTENSIONAHA.114.04915

15. Yao HM, Shen DL, Zhao XY, et al. Prognostic value of total bilirubin in patients with angina pectoris undergoing percutaneous coronary intervention. Int J Clin Exp Med. 2015;8(9):15930-15939.

16. Acet H, Erts F, Akil MA, et al. A novel predictor of infarct-related artery patency before percutaneous intervention and in-hospital outcomes for ST-segment elevation myocardial infarction patients: serum bilirubin level. Postep Kardiol Inter. 2014;10(2):91-97. doi:10.5114/pwki.2014.43513

17. Celik T, Kaya MG, Akpek M, et al. Does Serum Bilirubin level on admission predict TIMI flow grade and in-hospital MACE in patients with STEMI undergoing primary PCI. Angiology. 2014;65 (3):198-204. doi:10.1177/0003319712474948

18. Gul M, Uyarel H, Ergelen M, et al. Prognostic value of total bilirubin in patients with ST-segment elevation acute myocardial infarction undergoing primary coronary intervention. Am J Cardiol. 2013;111 (2):166-171. doi:10.1016/j.amjcard.2012.09.011

19. Huseynov A, Baumann S, Becher T, et al. Liver and cholestatic parameters as prognostic biomarkers of in-hospital MACE in patients with STEMI. Eur J Clin Invest. 2016;46(8):721-729. doi:10.1111/eci.12655
20. Wu AH, Levy WC, Welch KB, et al. Association between bilirubin and mode of death in severe systolic heart failure. Am J Cardiol. 2013;111(8):1192-1197. doi:10.1016/j.amjcard.2012.12.048

21. Frikha Z, Ferreira JP, Bozec E, et al. Relation of high serum bilirubin to short-term mortality following a myocardial infarction complicated by left ventricular systolic dysfunction (from the high-risk myocardial infarction database initiative). Am J Cardiol. 2018;121 (9):1015-1020. doi:10.1016/j.amjcard.2018.01.012

22. Yao HM, Wan YD, Zhang XJ, et al. Long-term follow-up results in patients undergoing percutaneous coronary intervention (PCI) with drug-eluting stents: results from a single high-volume PCI centre. BMJ Open. 2014;4(8):e004892. doi:10.1136/bmjopen-2014-004892

23. Yao H, Wan Y, Zhang X, et al. Data from: long-term follow-up results in patients undergoing percutaneous coronary intervention (PCI) with drug-eluting stents: results from a single high-volume PCI center. Dryad Digital Repository. 2014. doi:10.5061/dryad.13d31

24. Breimer LH, Mikhailidis DP. Is bilirubin a marker of vascular disease and/or cancer and is it a potential therapeutic target? Curr Pharm Des. 2011;17(33):3644-3655.

25. Kimm H, Yun JE, Jo J, Jee SH. Low serum bilirubin level as an independent predictor of stroke incidence: a prospective study in Korean men and women. Stroke. 2009;40(11):3422-3427. doi:10.1161/STROKEAHA.109.560649

26. Amor AJ, Ortega E, Perea V, et al. Relationship between total serum bilirubin levels and carotid and femoral atherosclerosis in familial dyslipidemia. Arter Thromb Vasc Biol. 2017;37(12):2356-2363. doi:10.1161/ATVBAHA.117.310071

27. Shen H, Zeng C, Wu X, Liu S, Chen X. Prognostic value of total bilirubin in patients with acute myocardial infarction: A meta-analysis. Medicine (Baltimore). 2019;98(3):e13920. doi:10.1097/MD.0000000000013920

28. Sahin O, Akpek M, Elcik D, et al. Bilirubin levels and the burden of coronary atherosclerosis in patients with STEMI. Angiology. 2013;64 (3):200-204. doi:10.1177/0003319712440619

29. Htun NM, Chen YC, Lim B, et al. Near-infrared autofluorescence induced by intraplaque hemorrhage and heme degradation as marker for high-risk atherosclerotic plaques. Nat Commun. 2017;8(1):75. doi:10.1038/s41467-017-00138-x

30. Mito S, Ozono R, Oshima T, et al. Myocardial protection against pressure overload in mice lacking Bach1, a transcriptional repressor of heme oxygenase-1. Hypertension. 2008;51(6):1570-1577. doi:10.1161/HYPERTENSIONAHA.107.102566

31. Okuhara K, Kisaka T, Ozono R, et al. Change in bilirubin level following acute myocardial infarction is an index for heme oxygenase activation. South Med J. 2010;103(9):876-881. doi:10.1097/ SMJ.0b013e3181 eac06a

32. Amanvermez R, Acar E, Gunay M, Baydın A, Yardan T, Bek Y. Hsp 70, hsCRP and oxidative stress in patients with acute coronary syndromes. Bosn J of Basic Med Sci. 2012;12(2):102-107. doi:10.17305/bjbms.2012.2508 


\section{Supplementary material}

Table SI In-hospital and follow-up outcomes in ACS and SA patients

\begin{tabular}{|c|c|c|c|c|c|c|}
\hline \multirow[t]{3}{*}{ Clinical outcomes } & \multicolumn{3}{|l|}{ ACS patients } & \multicolumn{3}{|l|}{ SA patients } \\
\hline & \multirow{2}{*}{$\begin{array}{l}\text { TB>0.60 } \mathrm{mg} / \mathrm{dL} \\
\mathrm{n}=798\end{array}$} & \multirow{2}{*}{$\begin{array}{l}\text { TB<=0.60 } \mathrm{mg} / \mathrm{dL} \\
\mathrm{n}=\mathrm{I}, 294\end{array}$} & \multirow[t]{2}{*}{$p$} & $\mathrm{~TB}>0.60 \mathrm{mg} / \mathrm{dL}$ & $T B<=0.60 \mathrm{mg} / \mathrm{dL}$ & \multirow[t]{2}{*}{$p$} \\
\hline & & & & $n=182$ & $\mathrm{n}=\mathbf{2 2 8}$ & \\
\hline \multicolumn{7}{|l|}{ In-hospital outcomes, n(\%) } \\
\hline Cardiovascular death & $9(1.1)$ & $7(0.5)$ & 0.134 & $2(I .1)$ & $0(0)$ & 0.113 \\
\hline Nonfatal MI & $5(0.6)$ & $6(0.5)$ & 0.617 & I $(0.5)$ & $3(1.3)$ & 0.432 \\
\hline MACE & $13(1.6)$ & $12(0.9)$ & 0.151 & $3(1.6)$ & $3(1.3)$ & $0.78 I$ \\
\hline \multicolumn{7}{|l|}{ Follow-up outcomes, n(\%) } \\
\hline MACE & $99(12.4)$ & $105(8.1)$ & 0.001 & $26(14.3)$ & $20(8.8)$ & 0.079 \\
\hline Cardiovascular death & $47(5.9)$ & $43(3.3)$ & 0.004 & $15(8.2)$ & $9(3.9)$ & 0.066 \\
\hline Nonfatal MI & $47(5.9)$ & $57(4.4)$ & 0.129 & $8(4.4)$ & $5(2.2)$ & 0.206 \\
\hline TVR & $43(5.4)$ & $54(4.2)$ & 0.152 & $14(7.7)$ & $12(5.3)$ & 0.316 \\
\hline Any revascularization & $67(8.4)$ & $99(7.7)$ & 0.528 & $18(9.9)$ & $15(6.6)$ & 0.221 \\
\hline In-stent restenosis & $49(6.1)$ & 7I (5.5) & 0.525 & $13(7.1)$ & $12(5.3)$ & 0.429 \\
\hline Angina & $96(12)$ & I44 (II.I) & 0.435 & $16(8.8)$ & $31(13.6)$ & 0.129 \\
\hline Stroke & $13(1.6)$ & $15(1.2)$ & 0.364 & $4(2.2)$ & $6(2.6)$ & 0.777 \\
\hline
\end{tabular}

Abbreviations: ACS, acute coronary syndrome; MACE, major adverse cardiovascular events; MI, myocardial infarction; SA, stable angina; TB, total bilirubin; TVR, target vessel revascularization.

\section{Publish your work in this journal}

Therapeutics and Clinical Risk Management is an international, peerreviewed journal of clinical therapeutics and risk management, focusing on concise rapid reporting of clinical studies in all therapeutic areas, outcomes, safety, and programs for the effective, safe, and sustained use of medicines. This journal is indexed on PubMed Central, CAS,
EMBase, Scopus and the Elsevier Bibliographic databases. The manuscript management system is completely online and includes a very quick and fair peer-review system, which is all easy to use. Visit http://www.dovepress.com/testimonials.php to read real quotes from published authors. 